\title{
Predicting sleep apnoea syndrome from heart period: a time-frequency wavelet analysis
}

\author{
F. Roche*, V. Pichot*, E. Sforza\#, I. Court-Fortune ", D. Duverney*, F. Costes*, M. Garet*, J-C. Barthélémy*
}

Predicting sleep apnoea syndrome from heart period: a time-frequency wavelet analysis. F. Roche, V. Pichot, E. Sforza, I. Court-Fortune, D. Duverney, F. Costes, M. Garet, J-C. Barthélémy. (C) ERS Journals Ltd 2003.

ABSTRACT: Heart rate fluctuations are a typical finding during obstructive sleep apnoea, characterised by bradycardia during the apnoeic phase and tachycardia at the restoration of ventilation. In this study, a time-frequency domain analysis of the nocturnal heart rate variability (HRV) was evaluated as the single diagnostic marker for obstructive sleep apnoea syndrome (OSAS).

The predictive accuracy of time-frequency HRV variables (wavelet (Wv) decomposition parameters from level 2 (Wv2) to level 256 (Wv256)) obtained from nocturnal electrocardiogram Holter monitoring were analysed in 147 consecutive patients aged $53.8 \pm 11.2$ yrs referred for possible OSAS.

OSAS was diagnosed in 66 patients $(44.9 \%)$ according to an apnoea/hypopnoea index $\geqslant 10$. Using receiver-operating characteristic curves analysis, the most powerful predictor variable was Wv32 (W $0.758, p<0.0001)$, followed by Wv16 (W 0.729, $\mathrm{p}<0.0001$ ) and Wv64 (W 0.700, p $<0.0001$ ). Classification and Regression Trees methodology generated a decision tree for OSAS prediction including all levels of Wv coefficients, from $\mathrm{Wv} 2$ to $\mathrm{Wv} 256$ with a sensitivity reaching $92.4 \%$ and a specificity of $90.1 \%$ (percentage of agreement $91.2 \%$ ) with this nonparametric analysis.

Time-frequency parameters calculated using wavelet transform and extracted from the nocturnal heart period analysis appeared as powerful tools for obstructive sleep apnoea syndrome diagnosis.

Eur Respir J 2003; 22: 937-942.
*Physiology Laboratory, PPEH Group \& Association SYNAPSE, and Pneumology Dept and Sleep Laboratory, CHU Nord, Faculté de Médecine Jacques Lisfranc, Université Jean Monnet, Saint-Etienne, France. \#Sleep Laboratory, Department of Psychiatry, University Hospital, Geneva, Switzerland.

Correspondence: F. Roche, Laboratoire de Physiologie, Groupe PPEH \& Association de Recherche SYNAPSE, Exploration Fonctionnelle Cardio-Respiratoire, CHU Nord - Niveau 6, F-42055 Saint-Etienne Cedex 2, France. Fax: 33477828447

E-mail: Frederic.Roche@univ-st-etienne.fr

Keywords: Autonomous nervous system activity

electrocardiogram holter monitoring heart rate variability

obstructive sleep apnoea syndrome

Received: November 172002

Accepted after revision: July 12003
Obstructive sleep apnoea syndrome (OSAS) is a common prevalent problem [1] (prevalence of $4 \%$ in middle-aged males) with major health implications ranging from traffic accident [2] to serious cardiac arrhythmias. OSAS is associated with increased risks of hypertension, myocardial infarction and stroke, and with increased mortality rates [3, 4].

Diagnosis of OSAS is usually performed by polysomnography in a sleep laboratory, consisting of the measurement and recording of several signals used to analyse sleep and breathing. Whereas polysomnography represented the "gold standard" for the diagnosis, it is an expensive and timeconsuming procedure with important resources invested in patients with mild-to-moderate disease. Moreover, the laboratory environment often disturbs or interferes with the patient's sleep. Therefore, several strategies have been developed to decrease the number of the sleep recordings, including sleep questionnaires, ambulatory recordings, simplified multichannel systems and nocturnal oximetry, all showing a high specificity but a low sensitivity [5-7].

It is known that all through the night, recurrence of apnoeas elicits a typical and cyclic heart rate pattern consisting of cyclical brady/tachycardia [8], contrasting with an altered diurnal control of the sinus node, and is related to cyclic changes in vagal and sympathetic activity. To quantify the unique heart rate rhythm induced by a successive alternance of vagal stimulation and sympathetic discharge $[9,10]$, spectral analysis of heart rate variability (HRV) has

For editorial comments see page 870. been applied using short-term night-time recording. However, such a technique encounters major difficulties like the nonstationary pattern of data, the complex definition of the appropriate spectral band to capture the pathological rhythm, and the requirement of interpolation and resampling prior to signal processing. To avoid the stationary requirements, the time-frequency method of wavelet (Wv) transform has been proposed [11]. From a physiological point of view, this method, applied to RR interval time series, indicates a more accurate adaptation to autonomic blockade and allows time localisation of this adaptation. Using classical time-domain analysis of HRV, the authors recently demonstrated that 24-h monitoring may be a useful tool to diagnose OSAS and to identify obstructive sleep apnoea during nonrapid eye movement (NREM) sleep [12].

The goal of this study was to assess whether a timefrequency method of Wv transform could yield essential information on the diagnosis of OSAS. For that purpose, the sensitivity and specificity of this methodology of HRV analysis was evaluated during a full night's electrocardiogram (ECG) recording, in a large group of unselected patients examined for possible sleep-related breathing disorders.

\section{Subjects and methods}

\section{Study group}

The population studied consisted of 147 patients (101 males and 46 females) with a mean $\pm \mathrm{SD}$ age of $53.8 \pm 11.2 \mathrm{yrs}$, 
referred to the university hospital for clinically suspected OSAS. Exclusion criteria were permanent or paroxysmal atrial fibrillation, diabetes mellitus, Shy-Drager syndrome, and permanent ventricular or atrial pacing; none of them received antiarrhythmic drugs or digitalis. The HRV analysis and the polygraphic scoring were done by two independent scorers, each one blinded to the results of the other.

\section{Sleep study and polysomnography scoring}

OSAS was diagnosed on the basis of clinical criteria and on the polysomnography performed, following the recommendations of the American Sleep Disorders Association [13]. The presence and stages of sleep were monitored using two pairs of electroencephalographic leads (C4-A1, O2-A1), two pairs of electro-oculographic leads and chin electromyographic leads. Airflow was measured by an oronasal thermocoupler. Respiratory efforts were monitored using inductive plethysmography, with transducers placed around the chest and the abdomen. Arterial oxygen saturation $\left(\mathrm{Sa}, \mathrm{O}_{2}\right)$ was recorded continuously by pulse oximetry (Criticare Systems Inc., Waukesha, WI, USA) during the whole night period. The polysomnogram was scored manually according to standard criteria [14, 15].

Respiratory events were scored using criteria proposed by the American Academy of Sleep Medicine. Hypopnoeas were defined as $a \geqslant 50 \%$ reduction in airflow from the baseline value lasting for $\geqslant 10 \mathrm{~s}$ and associated with a $3 \%$ desaturation or an arousal. Apnoeas were defined as the absence of oronasal airflow lasting for $>10 \mathrm{~s}$. Obstructive apnoea was defined as the absence of airflow for $>10 \mathrm{~s}$ in the presence of persistent respiratory efforts. The apnoea/hypopnoea index (AHI) was established as the ratio of the number of apnoeas and hypopnoeas per hour of sleep. As an index of nocturnal hypoxaemia, the total time with $\mathrm{Sa}, \mathrm{O}_{2}<90 \%$ was considered. AHI $\geqslant 10$ was chosen as the threshold to identify the presence of OSAS.

\section{Simultaneous electrocardiogram Holter monitoring and heart rate variability analysis}

The nocturnal recordings (duration $7.3 \pm 1.1 \mathrm{~h}$ ) were analysed on a Novacor system (Rueil-Malmaison, France) model, equipped with the HRV module. To perform the analysis, each QRS complex was validated and the length between each QRS (RR interval) calculated. Only normal-to-normal beats were considered for analysis with intervals excluded due to ectopy or artefacts being replaced by holding the previous coupling interval level throughout the time interval to the next valid coupling interval. The ECG Holter system allowed extracting the list of RR intervals with a precision of $1 / 200 \mathrm{~s}$. Then, a $2.4 \mathrm{~Hz}$ resampling process was applied.

\section{Wavelet analysis}

Unlike Fourier, Wv transform is devoted to the analysis of nonstationary signals $[16,17]$. Thus, there is no prerequisite regarding the stability of the frequency content along the signal analysed. This analysis allows the extraction of the characteristic frequencies contained along a signal, which, in this case, was composed of consecutive intervals between RR interval series. The decomposition of a signal by $\mathrm{Wv}_{v}$ transform requires an adequate regular and localised mother function. Starting from this initial function, a family of functions is built by dilatation and translocation, which constitutes the so-called Wv frame. The analysis consists of sliding a window of different weights (corresponding to different levels) containing the $\mathrm{Wv}$ function, all along the signal. The calculation gives a serial list of coefficients named Wv coefficients, which represent the evolution of the correlation between the signal $\mathrm{f}$ and the chosen $\mathrm{Wv}$ at different levels of analysis (or different ranges of frequencies) all along the signal $\mathrm{f}$. In this analysis, the Daubechies four Wv transform was used [18]. For each record, the $\mathrm{Wv}$ coefficients were calculated on sets of 512 RR intervals, giving eight separate levels of analysis named Wv2, Wv4, Wv8, Wv16, Wv32, Wv64, Wv128 and Wv256. Then, the variability power, level by level, was calculated as the sum of squares of the coefficients. Thus, for each recording, the variability power for each level was obtained. The sum of $\mathrm{Wv}$ power coefficients at levels 2, 4 and 8, approximately corresponds to the Fourier high frequencies (an index of parasympathetic activity), $\mathrm{Wv}$ power coefficients at levels 16 and 32 roughly corresponds to the Fourier low frequencies, and $\mathrm{Wv}$ power coefficients at levels 64 and 128 to the Fourier very low frequencies.

\section{Statistical analysis}

Differences were considered as significant for $\mathrm{p}<0.05$. Values were expressed as mean \pm SD. Statistical analyses were performed to evaluate the ability of the variables to discriminate between affected (OSAS+) and nonaffected (OSAS-) status. Thus, the dependent variable was the diseased state (OSAS+). The independent variables analysed were Wv levels from Wv2 to Wv256. Receiver-operating curve (ROC) analysis was used $[19,20]$, with the areas under the curves represented by the letter W. A W value of 0.5 means that the distributions of the variables are similar in both populations. Conversely, a $\mathrm{W}$ value of 1.0 means that the distributions of the variables of two populations do not overlap at all. Stepwise regression analysis was used to confirm the previously performed ROC curves analysis. The dependent variable was represented by the AHI and the independent variables included were the Wv levels, which demonstrated a significant $\mathrm{W}$ value with ROC curves.

A classification tree was then built using the discriminant variables as indicated by the ROC curves. The first variable used was the one determining the best separation between diseased and nondiseased subjects. The other variables were then introduced according to a descending order of discriminative capacity. For each continuous variable, the cutoff value acting as a separator to make the decision was chosen as that offering the smallest number of misclassification, i.e. that which minimises the sum of false-positive and false-negative results. Sensitivity, specificity and percentage of agreement were calculated on a learning sample of this last analysis. The principle of the Classification and Regression Trees (CART) method is to look at all possible splits for all variables included in the analysis i.e. the $\mathrm{Wv}$ levels. The results are in the form of an inverted tree. CART begins with a root node and, through a process of yes/no questions, generates descendant nodes. Some nodes are terminal, meaning that a final determination for classification is reached while other nodes continue to be split until terminal nodes are reached. The first topic addresses the method CART uses to select its questions for splitting nodes. The next activity is to rank order each splitting rule on the basis of a goodness-ofsplit criterion. Once a best split is found, CART repeats the search process for each child node, continuing recursively until further splitting is impossible or stopped. Then, CART proceeds by growing trees until it is not possible to grow them any further. It generates a maximal tree and a set of subtrees. Cross-validation was followed by dividing the learning sample 
into 10 approximately equal parts containing similar distributions for the dependent variable. The results of the 10 minitest samples were finally combined to form an error rate for trees of any possible size; these error rates are applied to the tree based on the entire learning sample. The outcome of this complex process is a set of fairly reliable estimates of the independent predictive accuracy of the tree.

\section{Results}

Table 1 summarises the clinical variables of the patient group. OSAS was diagnosed in 66 patients (44.9\%) using polysomnography recording. No significant differences in

Table 1.-Clinical characteristics of the population

\begin{tabular}{lccc}
\hline Clinical characteristics & OSAS+ & OSAS- & p-value \\
\hline Subjects n & 66 & 81 & \\
Age yrs & $55.8 \pm 11.2$ & $54.9 \pm 10.7$ & 0.54 \\
Male & $51(77.3)$ & $50(61.7)$ & 0.23 \\
$\mathrm{BMI} \mathrm{kg} \cdot \mathrm{m}^{-2}$ & $28.9 \pm 9.2$ & $29.9 \pm 9.9$ & 0.15 \\
$\mathrm{AHI} \mathrm{n} \cdot \mathrm{h}^{-1}$ & $39.0 \pm 19.5$ & $2.6 \pm 2.6$ & $<0.001$ \\
$\mathrm{Sa}_{\mathrm{a}} \mathrm{O}_{2} \%$ & $92.2 \pm 4.7$ & $95.2 \pm 2.8$ & $<0.001$ \\
$\mathrm{~S}_{\mathrm{a}, \mathrm{O}_{2}}<90 \%$ (\% of TST) & $23.8 \pm 9.2$ & $9.2 \pm 20.8$ & $<0.001$ \\
Hypertension & $25(37.9)$ & $21(25.9)$ & 0.15 \\
\hline
\end{tabular}

Data are presented as mean $\pm \mathrm{SD}$ or $\mathrm{n}(\%)$ unless otherwise stated. OSAS+: patients with obstructive sleep apnoea syndrome; OSAS-: patients without obstructive sleep apnoea syndrome; BMI: body mass index; AHI: apnoea/hypopnoea index; $\mathrm{Sa}, \mathrm{O}_{2}$ : arterial oxygen saturation; TST: total sleep time.
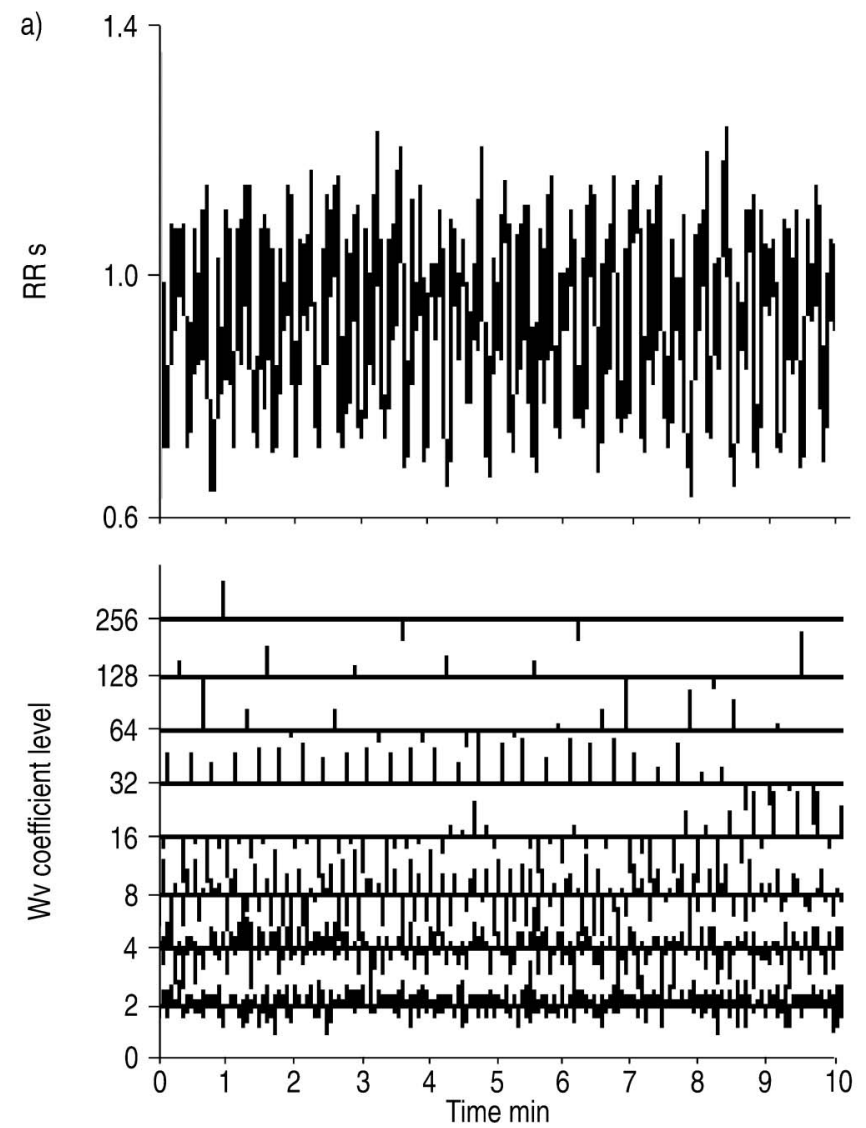

clinical characteristics, age and body mass index were found between OSAS+ and OSAS- patients.

Wv decomposition of the HRV analysis during the night in a typical OSAS patient (AHI 40) is illustrated in figure 1. With a subjective, first, visual approach, an enhancement of the coefficients for the levels Wv8, 16, 32, 64 and 128 can be noted, compared with a nonapnoeic patient.

ROC curves (continuous data) were built separately for each HRV variable (table 2). All time frequency-domain variables (Wv2 to Wv256) calculated over the full night's recording were able to separate OSAS+ from OSAS- status with statistical significance $(\mathrm{p}<0.05)$. Three variables appeared as outstanding separators: Wv32 (W 0.758, p<0.0001), Wv16 (W $0.729, \mathrm{p}<0.0001)$ and $\mathrm{Wv} 64$ (W 0.700, $\mathrm{p}<0.0001$ ).

Results of the optimal tree built using CART methodology are presented in table 3 . Figure 2 contains the essentials of the CART classification analysis. The classification tree diagram presents 20 terminal regions and 19 nodes of information. The Wv32 level represents the most important variable (first node) followed by Wv16, Wv8 and Wv64. Table 4 shows that the number of misclassifications is very low on the learning sample with a total number of eight false positives and of five false negatives (fig. 2). The sensitivity reached $92.4 \%$, the specificity $90.1 \%$ and the percentage of agreement was $91.2 \%$ in this population.

\section{Discussion}

The most important finding of this study was that the recurrence of obstructive apnoeas determines a significant

b)

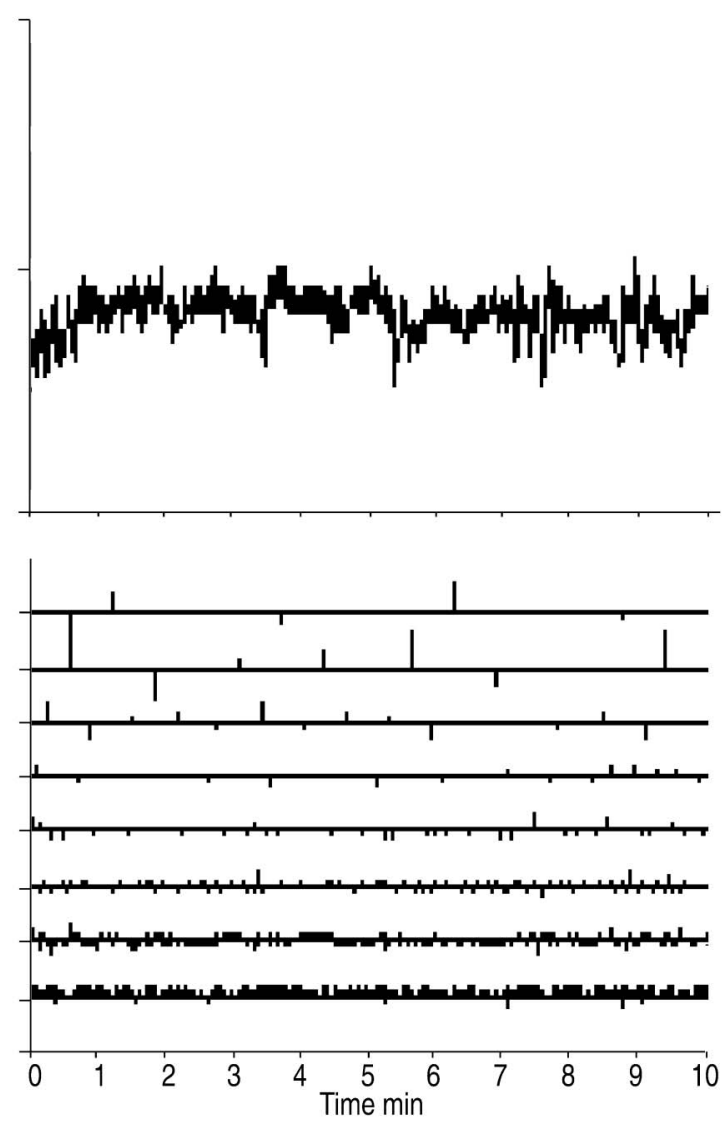

Fig. 1.-Wavelet (Wv) decomposition of the heart rate variability signal during the night in a) an obstructive sleep apnoea patient and b) a patient without sleep-related breathing disorders during a 10-min sample duration. 
Table 2. - Time frequency-domain variables significantly associated with obstructive sleep apnoea syndrome by receiveroperating characteristic curve analysis

\begin{tabular}{lcc}
\hline Variable & W value & p-value \\
\hline Wv2 & 0.595 & 0.040 \\
Wv4 & 0.609 & 0.025 \\
Wv8 & 0.661 & 0.0009 \\
Wv16 & 0.729 & $<0.0001$ \\
Wv32 & 0.758 & $<0.0001$ \\
Wv64 & 0.700 & $<0.0001$ \\
Wv128 & 0.642 & 0.0033 \\
Wv256 & 0.633 & 0.006 \\
\hline
\end{tabular}

Wv: wavelet power coefficients.

increase in the time-frequency components of the HRV. The alteration in the power coefficient of level 8, 16 and 32 was highly predictive of this sleep-related breathing disorder with a high sensitivity and specificity. The high prediction of OSAS based on the heart rate period analysis in these populations suggests that this methodology may be a potential simple and robust ambulatory screening method for diagnosis of OSAS patients [21].

Based on the typical oscillations in heart rate, firstly described by GUILLEMINAULT et al. [8], several devices have been used to diagnose OSAS, including short-term, timefrequency spectral analysis of the HRV shown as a diagnostically accurate method for detection of OSAS. Recent data
Table 3.-Relative importance of heart rate variability evaluated using Classification and Regression Trees methodology in order to predict obstructive sleep apnoea syndrome with an apnoea/hypopnoea index threshold value of 10

\begin{tabular}{lc}
\hline Variable & Relative importance $\%$ \\
\hline Wv32 & 100 \\
Wv16 & 78.10 \\
Wv8 & 73.02 \\
Wv64 & 70.21 \\
Wv128 & 68.86 \\
Wv4 & 58.26 \\
Wv256 & 58.09 \\
Wv2 & 36.60 \\
\hline
\end{tabular}

on time-domain measurements published by the present group clearly demonstrated that the analysis of the timedomain HRV variables is a useful tool in OSAS screening [12]. The accuracy of the present method was very high over 15-min selected periods of NREM sleep. In addition, the specificity and sensitivity were found to be significantly greater compared with published data using oximetry. Interestingly, SHIOMI et al. [9] demonstrated that this method is an easy tool not only for screening, but also for follow-up evaluation, showing a significant decrease in the very low-frequency component of HRV after mandibular advancement treatment. Despite its validity, standard HRV

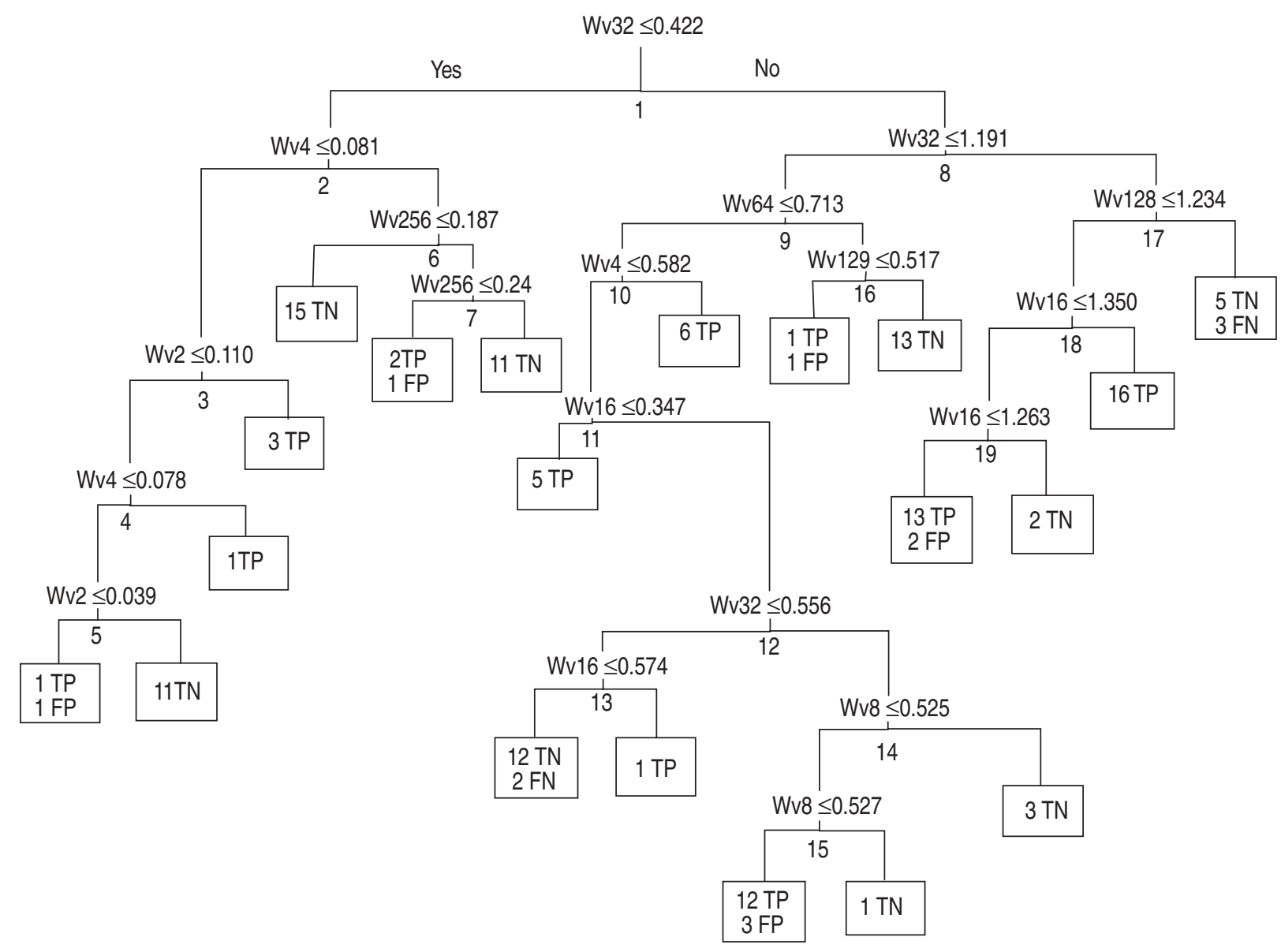

Fig. 2.- Operation tree illustrating the combination of threshold values of wavelet (Wv) levels, characterising patients in the study. Sensitivity reaches $92.4 \%$ and specificity $90.1 \%$. All individual numerical values are indicative of the number of subjects in each terminal node of the tree. TP: true positive, $n=61$; FP: false positive, $n=8 ; T N$ : true negative, $n=73 ; F N$ : false negative, $n=5$. 
Table 4. - Cross-validation and misclassification by class (obstructive sleep anoea syndrome (OSAS)+/-) using Classification and Regression Trees methodology

\begin{tabular}{|c|c|c|c|c|c|c|c|}
\hline \multirow[t]{2}{*}{ Class } & \multirow[t]{2}{*}{ Prior probability } & \multicolumn{3}{|c|}{ Cross-validation } & \multicolumn{3}{|c|}{ Learning sample } \\
\hline & & $\mathrm{n}$ & Misclassification $\mathrm{n}$ & Cost & $\mathrm{n}$ & Misclassification $\mathrm{n}$ & Cost \\
\hline OSAS- & 0.500 & 81 & 29 & 0.3580 & 81 & 8 & 0.0987 \\
\hline OSAS+ & 0.500 & 66 & 16 & 0.2424 & 66 & 5 & 0.7575 \\
\hline Total & 1.000 & 147 & 45 & & 147 & 13 & \\
\hline
\end{tabular}

Independent heart rate variables were Wv2, Wv4, Wv8, Wv16, Wv32, Wv64, Wv128 and Wv256. See text for more details.

frequency-domain analysis did not appear an appropriate tool to quantify variations in very low-frequency oscillations, a stronger marker of obstructive apnoea. For this reason, it was hypothesised that repetitive low-frequency oscillation, frequency-domain analysis of the heart period could be a better predictor of OSAS [22]. Compared with power spectral density of the very low frequency components (interbeat interval increment), $\mathrm{Wv}$ analysis gives a much higher specificity and also a higher sensitivity, without more intervention from the user. Applying the time-frequency analysis in a large group of unselected patients, this method was found to allow a better quantification of the frequency components associated with the obstructive apnoea events, with a highly discriminant power of three new variables discriminating patients with OSAS. Three levels of Wvs, Wv8, Wv16 and $\mathrm{Wv} 32$, are clearly predictive of the oscillations generated by the obstructive respiratory events. While the Wv8 level is usually considered as a parasympathetically mediated frequency [18], the Wv16 and Wv32 correspond to the lowfrequency component of the classical Fourier transform. If Wv32 represents the first node of the regression tree for an OSAS defined with an AHI of $>10$, Wv16 and Wv8 levels appear in the ROC curve analysis as further strong predictive variables in order to predict OSAS. It could seem surprising that very low-frequency components (Wv64, Wv128) do not represent the most appropriate spectral band to capture the brady/tachycardia rhythm. The discordance in these results could be explained by the observation by HiLTON et al. [11] that the low frequencies $(0.01875-0.07383 \mathrm{~Hz})$ correspond to the authors' Wv32 and Wv64 levels. From a practical point of view, an evaluation based on these three combined parameters, at least, should probably be proposed in a future prospective cohort of patients.

From the literature, $\mathrm{Sa}, \mathrm{O}_{2}$ bears the same sensitivity but a lower specificity than the present $\mathrm{Wv}$ analysis. Specificity using $\mathrm{Sa}_{2} \mathrm{O}_{2}$ ranges $46-80 \%[5,6]$. Furthermore, the authors did not intend to compare the sensitivity of $\mathrm{Wv}$ and $\mathrm{Sa}, \mathrm{O}_{2}$ in this study, since the cost-effectiveness of the nocturnal $\mathrm{Sa}_{2} \mathrm{O}_{2}$ analysis has already been largely described in sleep apnoea syndrome detection.

Certain limitations of this study that could influence the results need to be considered. The first consideration is that patients with autonomic disorders or arrhythmias were excluded to limit false-negative results. However, complete polygraphy also carry some limitations and oximetry could be misleading by being unable to identify nonapnoeic nocturnal hypoventilation, as well as to underestimate the severity of sleep-related breathing disorders. Secondly, patients suspected of having OSAS were predominantly studied despite them belonging to a heterogeneous group in terms of AHI. Therefore, the sample may not fully represent the general population of patients referred to clinicians, and those having snoring or upper airway resistance syndrome. However, STRADLING et al. [23], underlined that the autonomic arousals related to OSAS were better quantified using blood pressure variability [24, 25]. In addition, O'DonNELl et al. [26] demonstrate that digital vascular tone, measured by peripheral arterial tonometry, provides a reliable method to detect airway obstruction and arousal, reflecting the blood pressure and heart rate changes occurring during the upper airway obstruction.

Even though the methodology used in the present study may artificially increase the power of HRV analysis in OSAS patients, more extensive studies including patients with a wide range of sleep-disordered breathing could assess the validity and the diagnostic prediction of HRV analysis. Finally, the observed enhanced HRV found in these OSAS patients reflects the role of three factors, i.e. increase in negative oesophageal pressure, hypoxia and arousal occurring throughout and at the end of apnoea, all of which induce a strong increase in the parasympathetic system, followed by an abrupt sympathetic activation due to the consecutive hypoxia [27, 28]. These cyclical and constant responses could be compared with the primary slow oscillations in heart rate generated by the central nervous system during Cheyne-Stokes or periodic breathing patterns [29], probably reflecting a loop response of the cardiopulmonary chemo- and/or baroreceptors. For these considerations it would be interesting to examine patients with central apnoeas in order to determine whether the same Wv variables could predict the presence of altered respiration during sleep.

In conclusion, time-frequency domain analysis of the nocturnal heart rate variability using Wavelet decomposition could represent an efficient marker of obstructive sleep apnoea syndrome. Its added ease of use and of interpretation is of interest considering the high prevalence of sleep-related breathing disorders in a general middle-aged, at-risk population. Further, studies considering larger populations, with a wide spectrum of sleep-related breathing disorders, need to be performed in order to determine whether this method may be an easy tool to use for the diagnosis of the whole spectrum of sleep-related breathing disorders on an ambulatory basis.

\section{References}

1. Young T, Palta M, Dempsey J, Skatrud S, Weber S, Badr S. The occurrence of sleep-disordered breathing among middleaged adults. $N$ Engl J Med 1993; 328: 1230-1235.

2. Barbé F, Pericas J, Munoz A, Findley L, Anto JM, Agusti AGN. Automobile accidents in patients with sleep apnea syndrome. An epidemiological study. Am J Respir Crit Care Med 1998; 158: 18-22.

3. Lavie P, Peled R, Berger I, Yoff N, Zomer J, Rubin AH. Mortality in sleep apnea patients: a multivariate analysis of risk factors. Sleep 1995; 18: 149-157.

4. He J, Kryger MH, Zorick FJ, Conway W, Roth T. Mortality and apnea index in obstructive sleep apnea: experience in 385 male patients. Chest 1988; 94: 9-14.

5. Levy P, Pepin JL, Deschaux-Blanc C, Paramelle B, Brambilla C. 
Accuracy of oximetry for detection of respiratory disturbances in sleep apnea syndrome. Chest 1996; 109: 395-399.

6. Series F, Marc I, Cormier Y. Utility of nocturnal home oxymetry for case finding in patients with suspected sleep apnea hypopnea syndrome. Ann Intern Med 1993; 119: 449-453.

7. Epstein LJ, Dorlac GR. Cost-effectiveness analysis of nocturnal oximetry as a method of screening for sleep apnea-hypopnea syndrome. Chest 1998; 113: 97-103.

8. Guilleminault C, Connoly S, Winkle R, Melvin K, Tilkian A. Cyclical variation of the heart rate in sleep apnoea syndrome. Lancet 1984; 21: 126-131.

9. Shiomi T, Guilleminault C, Sasanabe R, Hirota I, Maekawa M, Koboyashi T. Augmented very low frequency component of heart rate variability during obstructive sleep apnea. Sleep 1996; 19: 370-377.

10. Keyl C, Lemberger P, Pfeifer M, Hochmuth K, Geisler P. Heart rate variability in patients with daytime sleepiness suspected of having sleep apnoea syndrome: a receiveroperating characteristic analysis. Clin Sci 1997; 92: 335-343.

11. Hilton MF, Bates RA, Godfrey KR, Chappell MJ, Cayton RM. Evaluation of frequency and time-frequency spectral analysis of heart rate variability as a diagnostic marker of the sleep apnoea syndrome. Med Biol Eng Comput 1999; 37: 760-769.

12. Roche F, Gaspoz JM, Court-Fortune I, et al. Screening of obstructive sleep apnea syndrome by heart rate variability analysis. Circulation 1999; 100: 1411-1415.

13. Thorpy MJ, and the Diagnostic Classification Steering Committee. The International Classification of Sleep Disorders: Diagnostic and Coding Manuel. Rochester, American Sleep Disorders Association, 1990; pp. 52-58.

14. American Academy of Sleep Medecine. Sleep-related breathing disorders in adults: recommendation for syndrome definition and measurement techniques in clinical research. Sleep 1999; 22: 667-689.

15. Rechtschaffen A, Kales A, eds. A Manual of Standardized Techniques and Scoring Systems for Sleep Stages of Human Subjects. Publication No. 204. Washington, National Institutes for Health, 1968.

16. Task Force of the European Society of Cardiology and the North American Society of Pacing and Electrophysiology. Heart rate variability: standards of measurements, physiological interpretation, and clinical use. Circulation 1996; 93: $1043-1065$.
17. Akay M. Introduction: Wavelet transforms in biomedical engineering. Ann Biomed Eng 1995; 23: 529-530.

18. Pichot V, Gaspoz JM, Molliex S, et al. Wavelet transform to quantify heart rate variability and to assess its instantaneous changes. J Appl Physiol 1999; 86: 1081-1091.

19. Hanley JA, McNeil BJ. The meaning and use of the area under receiver operating characteristic (ROC) curve. Radiology 1982; 143: 29-36.

20. Hanley JA, McNeil BJ. A method of comparing the areas under receiver operating characteristic curves derived from the same cases. Radiology 1983; 148: 839-843.

21. Goldberger AL, Amaral LAN, Glass L, et al. PhysioBank, PhysioToolkit, and PhysioNet. Components of a new research resource for complex physiologic signals. Circulation 2000; 101: e215-e220.

22. Roche F, Duverney D, Court-Fortune I, et al. Cardiac interbeat interval increment for the identification of obstructive sleep apnea. Pacing Clin Electrophysiol 2002; 25: 11921199.

23. Stradling JR, Davies RJO, Pitson DJ. New approaches to monitoring sleep-related breathing disorders. Sleep 1996; 19: S77-S84

24. Pitson DJ, Stradling JR. Value of beat-to-beat pressure changes, detected by pulse transit time, in the management of the obstructive sleep apnoea/hypopnoea syndrome. Eur Respir J 1998; 12: 685-692.

25. Pitson DJ, Stradling JR. Autonomic markers of arousal during sleep in patients undergoing investigation for obstructive sleep apnoea, their relationship to EEG arousals, respiratory events and subjective sleepiness. J Sleep Res 1998; 7: 53-59.

26. O'Donnell CP, Allan L, Atkinson P, Schwartz AR. The effect of upper airway obstruction and arousal on peripheral arterial tonometry in obstructive sleep apnea. Am J Respir Crit Care Med 2002; 166: 965-971.

27. Somers VK, Dyken ME, Clary MP. Sympathetic neural mechanisms in obstructive sleep apnea. J Clin Invest 1995; 96: 1897-1904.

28. Leung ST, Bradley D. Sleep apnea and cardiovascular disease. Am J Respir Crit Care Med 2001; 164: 2147-2165.

29. Ichimaru Y, Yanaga T. Frequency characteristics of the heart rate variability produced by Cheyne-Stokes respiration during 24-hour ambulatory electrocardiographic monitoring. IEEE Comput Biomed Res 1989; 22: 225-233. 